\title{
Hope and Hype of Retinal Vascular Imaging in Cerebral Small Vessel Disease
}

\author{
Michele Cavallari ${ }^{1}$ and Francesco Orzi ${ }^{2 *}$ \\ ${ }^{1}$ Center for Neurological Imaging, Department of Radiology, Brigham and Women's Hospital, Harvard Medical School, Italy \\ ${ }^{2}$ NESMOS Department, University of Rome "La Sapienza", Rome, Italy
}

"Corresponding author: Francesco Orzi, Department of Neurosciences, Mental Health and Sense Organs (NESMOS), University of Rome "La Sapienza", Rome, Italy, Tel: +39 063377 5829; E-mail: francesco.orzi@uniroma1.it

Received date: October 07, 2015; Accepted date: October 9, 2015; Published date: October 16, 2015

Copyright: $@ 2015$ Orzi F et al. This is an open-access article distributed under the terms of the Creative Commons Attribution License, which permits unrestricted use, distribution, and reproduction in any medium, provided the original author and source are credited.

\section{Editorial}

Cerebral small vessel disease (SVD) refers to a broad spectrum of disorders, which share magnetic resonance imaging (MRI)-evident abnormalities, such as white matter hyperintensities (WMH), lacunes, microbleeds, and enlarged perivascular spaces [1]. The clinical phenotype of SVD is consistently represented by cognitive deficits, which mostly pertain to executive functions, attention or set-shifting abilities [2]. Sporadic, age-related SVD is associated with traditional vascular risk factors, especially hypertension and diabetes [3].

Clinical presentations and slopes of disease progression, as well as their histopathological correlates, are extremely heterogeneous $[4,5]$. The observed heterogeneity suggests that SVD encompasses a spectrum of disorders, which reflect vascular and neurodegenerative mechanisms. Confluent WMH and lacunes are considered secondary to endothelial dysfunction and chronic hypoperfusion [5,6]. Nonvascular mechanisms may also contribute to the development and evolution of these abnormalities, alternatively or in addition to perfusion changes. For instance, disruption of the ependymal lining with subependymal widening of the extracellular space [5], enlargement of perivascular space [7], changes in blood-brain barrier permeability [3] and cerebrospinal fluid content of beta-amyloid [8] are associated with development or expansion of WMH. All these phenomena are thought not directly caused by dysregulation of cerebral blood flow. It is, therefore, reasonable to assume that SVD includes different disease subtypes, potentially reflecting different pathogenic mechanisms, each susceptible of specific treatments. In the quest of stratification of subjects with SVD according to the predominant etiological mechanism/s, multimodal diagnostic approaches based on cerebrospinal fluid, neuropsychological and neuroimaging tools are being proposed [3].

Recent interest has emerged in analysis of the retinal vessels as an indicator of microvascular damage. Retinal vessels are thought to mirror the brain microvasculature [9] and morphological analysis by fundoscopy provides an inexpensive, immediate and non-invasive means for detecting and staging systemic microvascular damage. Quantitative methods [10,11] have recently overcome previous observer-driven approaches and provided us with a full exploitation of fundoscopy. There is evidence that morphometric parameters of retinal vessel geometry, such as vessel diameter, tortuosity, and fractal dimension, are altered in a number of conditions typically associated with SVD, including $\mathrm{WMH}$, microbleeds, lacunes, and cognitive decline [12]. Retinal changes were also observed in lacunar stroke subtype and, more significantly, in cerebral autosomal dominant arteriopathy with subcortical infarcts and leukoencephalopathy (CADASIL) $[11,13]$. CADASIL, the most common inherited SVD, is typically not associated with vascular risk factors. These observations, altogether, endorse quantitative retinal imaging as a tool for risk assessment, monitoring and possibly stratifying SVD.

Although the role of retinal imaging in stratifying individuals with different subtypes of SVD has been advocated, the hypothesis has never been formally tested. One of the major methodological challenges is the lack of a gold standard for defining the hypothetical subgroups of SVD. Our own preliminary results of a small study of 50 individuals with SVD suggest heterogeneous distribution of retinal vessel abnormalities among people with $\mathrm{WMH}$ and mild cognitive impairment (unpublished data). The apparent heterogeneity is consistent with the hypothesis of different pathogenic mechanisms underlying SVD. Since retinal vessel abnormalities are associated with both MRI correlates and established risk factors of SVD (such as diabetes and hypertension), it remains unclear whether the observed retinal alterations reflect primarily the brain microvessel abnormalities, or rather represent retinal damage associated with the vascular risk factors.

Another related challenge, which has implications in the implementation of retinal imaging in clinical settings, regards the effect size of the observed association between SVD and retinal vessel abnormalities, especially in longitudinal studies [12]. The association with conditions characterized by established vascular pathology (e.g., CADASIL) appears to become evident even in small samples [11,13]. The observed association with sporadic, age-related SVD seems weak, as a probable expression of the abovementioned heterogeneity.

Quantitative retinal imaging is a useful tool to assess risk of development and progression of SVD at the population level. Technical developments may likely improve specificity and enable stratification of subjects according to the predominant etiological mechanisms of different SVD subtypes.

\section{References}

1. JM Wardlaw, C Smith, M Dichgans (2013) "Mechanisms of sporadic cerebral small vessel disease: insights from neuroimaging," Lancet Neurol 12: 483-97.

2. L Pantoni (2010) "Cerebral small vessel disease: from pathogenesis and clinical characteristics to therapeutic challenges," Lancet Neurol 9: 689-701.

3. GA Rosenberg, A Wallin, JM Wardlaw, HS Markus, J Montaner et al. (2015) "Consensus statement for diagnosis of subcortical small vessel disease," J. Cereb. Blood Flow Metab.

4. Brickman AM, Siedlecki KL, Muraskin J, Manly JJ, Luchsinger JA et al. (2011) "White matter hyperintensities and cognition: testing the reserve hypothesis.," Neurobiol. Aging 32: 1588-1598.

5. Schmidt R, Schmidt H, Haybaeck J, Loitfelder M, Weis S et al. (2011) "Heterogeneity in age-related white matter changes.," Acta Neuropathol 122: $171-185$. 
Citation: Michele Cavallari and Francesco Orzi (2015) Hope and Hype of Retinal Vascular Imaging in Cerebral Small Vessel Disease. J Neurol Neurophysiol 6: e116. doi:10.4172/2155-9562.1000e116

Page 2 of 2

6. Fernando MS, Simpson JE, Matthews F, Brayne C, Lewis CE et al. (2006) "White matter lesions in an unselected cohort of the elderly: molecular pathology suggests origin from chronic hypoperfusion injury," Stroke 37: $1391-1398$.

7. GM Potter, FN Doubal, CA Jackson, FM Chappell, CL Sudlow et al. (2015) "Enlarged perivascular spaces and cerebral small vessel disease." Int. J. Stroke 10: 376-381

8. MI Kester, JD C Goos, CE Teunissen, MR Benedictus, FH Bouwman, et al. (2014) "Associations Between Cerebral Small-Vessel Disease and Alzheimer Disease Pathology as Measured by Cerebrospinal Fluid Biomarkers," JAMA Neurol., 71: 855-862.

9. N Patton, T Aslam, T MacGillivray, A Pattie, IJ Deary et al. (2005) "Retinal vascular image analysis as a potential screening tool for cerebrovascular disease: a rationale based on homology between cerebral and retinal microvasculatures," J. Anat 206: 319-348.
10. TY Wong (2004) "Is retinal photography useful in the measurement of stroke risk?” Lancet Neurol 3: 179-183.

11. M Cavallari, C Stamile, R Umeton, F Calimeri, F Orzi (2015) "Nove Method for Automated Analysis of Retinal Images: Results in Subjects with Hypertensive Retinopathy and CADASIL," Biomed Res. Int., 752957.

12. Heringa SM, Bouvy WH, van den Berg E, Moll AC, Kappelle LJ, Biessels G (2013) Associations between retinal microvascular changes and dementia, cognitive functioning, and brain imaging abnormalities: a systematic review. J Cereb Blood Flow Metab 33: 983-995.

13. Cavallari M, Falco T, Frontali M, Romano S, Bagnato F et al. (2011) Fractal Analysis Reveals Reduced Complexity of Retinal Vessels in CADASIL. PLoS ONE 6: e19150 